\title{
Decálogo del ambientalismo estéril
}

\author{
ROBERTO J. FERNÁNDEZ \\ Cátedra de Ecología e IFEVA, Facultad de Agronomía, Universidad de Buenos Aires - CONICET, Argentina
}

[Palabras clave: buenas prácticas, capital social, catastrofismo, cientificismo, corrupción, ecologismo, globalización, multidisciplinariedad, tecnocracia, tragedia de los comunes]

[Keywords: best practices, corruption, crying wolf, globalization, multidisciplinarity, policy, scientism, technocracy, tragedy of the commons, social capital]

Toda verdad pasa por tres etapas: primero es ridiculizada; luego es resistida violentamente; más tarde es aceptada como una obviedad - atribuido a Schopenhauer

Existe acuerdo en la comunidad científica en que los problemas del planeta son muy serios. Hay datos confiables y alarmantes sobre el consumo no sustentable de energía, la pérdida de biodiversidad y los cambios acelerados en los ciclos de materiales (Rockström et al. 2009). Los medios de comunicación se ocupan frecuentemente de estos temas, sobre todo cuando hay protestas sociales relacionadas con ellos (e.g., pasteras, cultivos transgénicos y la minería a cielo abierto en Argentina), pero muchas veces lo hacen con información incorrecta o incompleta. En respuesta a esto, la Asociación Argentina de Ecología creó en 2009 la Comisión de Análisis de Temas Ambientales, de la que el autor de este artículo participó brevemente, y en la que no se pudo alcanzar acuerdos mínimos que permitieran instrumentar avances concretos. Algo similar había sucedido con iniciativas similares en la Asociación Española de Ecología terrestre, la Society for Conservation Biology, y la British Ecological Society (García Fayos et al. 2008).

Es preocupante que quienes nos dedicamos a algún aspecto de la ecología como principal actividad no podamos discutir con más claridad cuál debería ser nuestro papel. Por ejemplo, algo tan simple como si debe ser distinto del resto de los profesionales, científicos, o ciudadanos (Gurvich et al. 2009; Paruelo 2009). En particular quienes ejercemos la docencia, debemos cuidarnos de no transmitir mensajes excesivamente optimistas acerca de las posibilidades de que la situación ambiental mejore rápidamente, sin caer tampoco en el extremo de alentar el pesimismo y el descreimiento. Más conocimiento implica más responsabilidad, y nuestro trabajo incluye brindar a la sociedad más y mejor información, pero sobre todo esquemas lógicos y teóricos que sirvan como instrumentos para entender el mundo.

En lo que sigue se presenta una lista de diez afirmaciones que, a mi juicio, contienen la mayor parte de los obstáculos mentales para un mejor planteo de la situación ambiental. Aspiro a que esta lista sirva para dos propósitos: por un lado, para entender mejor por qué es tan difícil encarar los muchos y muy necesarios debates sobre el tema; por el otro para proveer ejes de análisis (o por lo menos disparadores) para esos debates, comenzando por nosotros como especialistas y docentes.

\section{Un DecÁlogo}

Propongo que la falta de respuesta a importantes mensajes relacionados con el ambiente por parte de la sociedad en general (y los niveles decisorios en particular) se debe en buena medida a posiciones comunicacionales y actitudinales incluidas en los diez puntos siguientes (Tabla 1). Elegí un formato irónico para aprovechar sus ventajas retóricas, pero además porque me parece que tenemos mucho más claro lo que no funciona que lo que sí. Un artículo como éste, de un solo autor y por lo tanto desde una sola disciplina y con

Editora asociada: Ana Cingolani 
una mirada inevitablemente influenciada por sesgos personales (ver punto \#5), jamás podría proponer la solución para las cuestiones ambientales (\#1, \#2). Aún para los problemas de menor escala, es necesario trabajo interdisciplinario (\#3), que luego tiene que ser debatido pública (\#9) y frecuentemente (\#10), y para el que no hay una metodología única que asegure el éxito en todos los casos, ya que ésta también debe ser consensuada (\#8). Este Decálogo intenta ser una "hoja de ruta" para encauzar las discusiones académicas, que son urgentes para definir las prioridades de investigación y para informar a la ciudadanía y mejorar los indispensables intercambios con la dirigencia (\#5-6).

\section{Creer, o actuar como si se creyese, que los problemas ambientales son técnicos, del dominio de una sola disciplina - muy probablemente la propia.}

Hace poco, para un congreso profesional, co-organicé un simposio sobre el llamado Paradigma de Zonas Áridas, uno de cuyos postulados más importantes es que para comprender la desertización es esencial considerar simultáneamente aspectos biofísicos y socioeconómicos (Reynolds et al. 2007). Los agrónomos y ecólogos a cargo del simposio veníamos trabajando desde hacía más de diez años sobre esas ideas. Así y todo, no incluimos en el panel expertos del área socioeconómica ni humanística, y no fuimos conscientes de esa omisión hasta que alguien del público lo mencionó. Claramente, actuamos como si pensásemos que lo único que importaba era nuestra área del conocimiento.

Como lo ha analizado profundamente Becher (1991), pertenecemos a "tribus" académicas, que actúan como si cada una fuese más importante que las demás. El caso personal relatado más arriba ni siquiera fue original en el contexto de las ciencias ambientales: Folke et al. (2005) han destacado que, pese a la toma de conciencia sobre la necesidad de esfuerzos de colaboración participativa (ver \#3,\#9), persiste la tendencia a que los científicos lleguen primero a sus conclusiones (y las agencias gubernamentales elaboren primero suagenda), para luego comunicarlas a los diferentes grupos de la comunidad, con el objetivo de incluirlos en el marco propuesto. Existen esfuerzos inter- y trans-disciplinarios serios, en algunos casos exitosos, pero como veremos en \#3 y \#8-9, ni surgen espontáneamente ni carecen de dificultades.

\section{Creer, o actuar como si se creyese, que las soluciones a los problemas ambientales son independientes del contexto político, económico y social.}

Esta visión es coherente con la del punto anterior, y de hecho tomadas juntas conducen al error de suponer que las tecnocracias serían viables, ya que estarían en condiciones de identificar y aplicar la mejor solución. SiguiendoaGeorges Clemenceau (quien habría dicho que "La guerra es un asunto demasiado serio para dejarlo en manos de los militares"), los temas ambientales nos afectan a todos, y son demasiado dependientes de los objetivos $\mathrm{y}$ valores que tengamos como sociedad como para delegarlos en los expertos-menos aún en los de una sola disciplina. Piénsese si no en las discusiones sobre el uso de energía en relación al cambio climático o cualquier tema que roce al crecimiento poblacional.

Pretender que las soluciones técnicas de las ciencias ligadas al ambiente puedan dirimir estos conflictos es como creer que la ciencia médica puede dar soluciones a todos los problemas de la salud pública. Los datos tomados aisladamente casi nunca son suficientes para modificar un curso de acción (de otro modo no habría médicos que fumen). Por el contrario, muchas veces la nueva información no ayuda a tomar una decisión sino más bien a demorarla, ya que es casi seguro que surgirán quienes aporten evidencias aparentemente válidas en la dirección opuesta (Sarewitz 2004), o que esgriman las incertidumbres como argumentos para una postergación (\#5; Jobbágy \& Fernández 2012). Esto de ningún modo quiere decir que la información científico-técnica sea irrelevante ni tampoco que la definición de las agendas de investigación deba dejarse librada al azar.

3. Creer, o actuar como si se creyese, que los mayores obstáculos a la aplicación de las soluciones a los problemas ambientales son el desconocimiento y la falta de honestidad.

Esta creencia es en parte consecuencia de las dos anteriores, ya que si las soluciones son relativamente simples sólo habría dos motivos por el que no se aplican: o la información no llega a quienes están encargados de tomar decisiones, o para ellos es beneficioso mantener el statu quo. En este último caso surge la sospecha de que la información no es buscada, y genera la repetida acusación de 
"falta de voluntad política". Sin embargo, esta posición da por sentado que el conocimiento adecuado ya existe. De ser así, deberá trabajarse sobre la comunicación (por ejemplo, a través de Parques Tecnológicos). Pero es común que la oferta de conocimiento no satisfaga las demandas de los potenciales usuarios (McNie 2007; Sarkki et al. 2013).

Lo que no hay que perder de vista es que el proceso que lleva de la ciencia básica a la aplicada y de ella a la tecnología no es lineal ni espontáneo por varios motivos, el más importante de los cuales es que no existen avances tecnológicos neutros: siempre se hacen para algo y para alguien. El desarrollo de la bomba atómica no emergió como una consecuencia natural de la nueva información sobre la estructura del átomo, sino que requirió una infraestructura equivalente a la de la industria automotriz norteamericana de la época (Rhodes 1987). En Argentina, como en otros países, es mucho lo que se ha escrito sobre las relaciones entre la política científica y el desarrollo económico-social (e.g., Varsavsky 1969; Sábato 2011).

Para resolver la desconexión entre oferta y demanda de información, Gibbons et al. (1994) propusieron convocar equipos multidisciplinarios, incluyendo representantes de la comunidad e idóneos, independientemente de sus calificaciones técnicas formales, por periodos cortos para generar ("co-producir") conocimiento directamente aplicable. Estas metodologías buscan conocimiento de Modo 2 (Gibbons et al. 1994), en contraposición al tradicional (Modo 1), que tiene objetivos ligados a los intereses académicos del investigador, está centrado en una disciplina, y no se preocupa por su aplicabilidad inmediata. Así y todo, es raro encontrar un investigador que no afirme que su línea de trabajo será eventualmente útil, por lo que sin una evaluación especialmente concebida no es fácildistinguirentreinformación relacionada con un problema y la más directamente relevante para su solución (e.g., Sarkki et al. 2013). El Ministerio de Ciencia, Tecnología e Innovación Productiva argentino ha creado recientemente un Banco Nacional de Proyectos de Desarrollo Tecnológico y Social con el objetivo de darle visibilidad al conocimiento más directamente aplicable y fomentar su generación.

\section{Creer, o actuar como si se creyese, que las soluciones propuestas no generan sus propios problemas ni tienen costos.}

La llamada Tragedia de los Comunes (Hardin 1968) plantea que los recursos deben ser protegidos de las acciones humanas destructivas, que sin embargo son totalmente racionales. Por lo tanto, cualquier propuesta de cambio debe partir de que existen costos de oportunidad como el dinero que dejarían de percibir algunas personas o grupos al no responderse estrictamente a la lógica del mercado. Está claro que algunas de estas personas y grupos suelen ser mucho más poderosos que la mayoría de los que se beneficiarían con el cambio (Bauman 2011), pero eso no descarta la existencia de otros que también puedan verse afectados en uno u otro sentido. Toda propuesta debería explicitar tanto sus costos como sus beneficios para los distintos grupos, porque las panaceas no existen.

Los llamados a modificar cualquier aspecto de la lógica dominante suelen ser respondidos con la acusación de que el énfasis en el cuidado ambiental desconoce las ventajas de los incentivos a la inversión para la creación de empleos y riqueza. Esto último es, como mínimo, una sobresimplificación que ignora la diversidad de visiones de los economistas sobre el tema (Román 2013) -tan errónea como la de confundir automáticamente a los ambientalistas con personas que se oponen al sistema capitalista.

5. Creer, o actuar como si se creyese, que sólo los adversarios usan selectivamente la información y están influenciados por intereses y sesgos ideológicos.

Hay quienes opinan que la ciencia debe mantenerse pura, separada de la política; otros, que debe usarse para enfrentar las decisiones más difíciles de la sociedad (Sábato 2011). La separación estricta no es posible, y es muy difícil que los asesores limiten su consejo a cuestiones técnicas sin que sus intereses y valores interfieran en la toma de posiciones objetivas (Jasanoff 1990). Un marco de referencia importante para entender estos temas es el que enfatiza el papel de los grupos de interés (advocacy coalitions, que pueden incluir científicos, técnicos, funcionarios, periodistas, empresas y hasta alianzas internacionales) como actores importantes en la definición de políticas públicas. Los miembros de estas coaliciones 
aceptarán inmediatamente toda evidencia a favor de sus posiciones e intereses, e ignorarán o cuestionarán toda evidencia en contrario (e.g. Sabatier y Zafonte 2001).

Estos "diálogos de sordos" se dan a todas las escalas, desde la definición del arbolado de una avenida hasta las cuestiones relacionadas con el cambio climático. Por eso, las disputas más serias sólo podrán resolverse en el marco de foros suficientemente prestigiosos, o con la intervención de brókers capaces de mantener posturas aceptablemente neutrales a los ojos de los intereses antagónicos (Folke et al. 2005). Cuando los científicos y técnicos son consultados, lo deseable es que propongan opciones de solución, más que una solución en particular, es decir que actúen como brókers de alternativas (Pielke 2007). Lo que nunca debe aceptarse es ni el lobby ni el activismo encubierto que tienen en común el presentar un curso de acción como el único favorecido por la objetividad científica (Jobbágy \& Fernández 2012). Como mínimo, la posibilidad de acuerdos existe mientras se comparta que hay métodos más aceptables que otros para aproximarse a la objetividad - métodos más científicos.

Sin embargo, cuando se enfrentan prioridades y escalas de valores diferentes, es fácil caer en por lo menos dos tipos de falacias: (i) el argumento ad consequentiam, i.e. concluir que una premisa es verdadera porque conduce a una consecuencia deseable (o, recíprocamente, falsa porque conduce a una indeseable), y en particular (ii) el ad hominem, dar por sentada la falsedad de una afirmación basándose en la falta de credibilidad de quien la enuncia. Un ejemplo para esta última: al recomendarle a un colega un video de David Attenborough para usar en clase, porque a mi juicio planteaba muy bien el delicadísimo tema del crecimiento poblacional, me dijo con toda honestidad que se negaba a verlo porque creía que los del Norte no tienen autoridad para hablar de ese tema, culpándonos por los impactos globales a los del Sur, cuando ellos son los que con su consumismo generan los mayores problemas.

6. Ignorar, o actuar como si se ignorase, el efecto contraproducente que pueden tener los mensajes catastróficos y repetitivos que anestesian a la opinión pública, alejándola de su necesario papel de aliada.

Jackson (2002) propuso que uno de los motivos por los que la tendencia declinante del ozono estratosférico pudo ser revertida con éxito fue que el problema apareció en la percepción pública de un modo más o menos súbito. En cambio, como en la fábula de Esopo, hemos estado escuchando "¡lobo, lobo!" por el cambio climático, el aumento de la superficie cultivada intensivamente y la pérdida de biodiversidad sin catástrofes que pudiesen ser unívocamente atribuidos a ellos, llevando a muchos a pensar, erróneamente, que no se trata de problemas reales. A diferencia del pastorcito mentiroso de la fábula la base de las denuncias es muchas veces cierta, pero el tono sensacionalista y poco específico del mensaje termina causando rechazo. La gente tiende a solidarizarse con el sufrimiento de los demás cuando es agudo, pero se acostumbra y paulatinamente deja de percibirlo si se vuelve crónico (Bauman 2011).

Una versión de catastrofismo muy relacionada con los sesgos mencionados en \#5 es el uso de calificativos despectivos o claramente insultantes (e.g., para la mayoría de los agrónomos, agrotóxico como sinónimo de agroquímico). Esta terminología subraya la posición de quien la emplea, pero también contribuye a la confusión, sobre-simplificando los problemas. Los fertilizantes son tan agroquímicos como los pesticidas, es decir los herbicidas, insecticidas y fungicidas, dentro de los cuales a su vez hay una enorme variación en toxicidad. Naturalmente, la credibilidad de los usuarios de una y otra versión del término queda instantáneamente dañada para los de la versión opuesta (falacia ad hominem).

\section{Ignorar, o actuar como si se ignorase, a la} globalización como una fuerza potentísima,
que si se desprecia nunca podrá ser usada a
favor.

La abundancia de recursos naturales (petróleo, minerales, tierra) que dan lugar a commodities exportables ha sido considerada una maldición para los países que los poseen (la Paradoja de la Abundancia), ya que tiende a obstaculizar su industrialización, muchas veces acrecentando las desigualdades internas en la distribución de ingresos (e.g., Williamson 2011). Esto se conoce como el Efecto Holandés, y proviene de la crisis desatada en las industrias de ese país a partir del descubrimiento, en 1959, de enormes reservas de gas natural. El petróleo venezolano y los diamantes del Congo y Angola son otros ejemplos más recientes bien conocidos y típicos, ya que ocurren en los países que Raúl 
Prebisch (1950) denominó de La Periferia (el Sur en sentido económico), noción más recientemente profundizada por Bauman (2011) desde su perspectiva de los Daños Colaterales, incluyendo los ambientales.

La reciente expansión en América Latina de la minería y el monocultivo extensivo, como la soja, son el resultado de tendencias similares a veces enmarcadas dentro del Neo-Extractivismo (Svampa 2013), pero no son en absoluto fenómenos nuevos (Ferrer 1997). En Argentina, los antecedentes fueron durante el siglo XIX la exportación de lana, carnes saladas y luego congeladas, y de metales preciosos durante el virreinato y los siglos anteriores. Ya entonces, las actividades relacionadas con la producción de esos bienes competitivos en el mercado internacional, y los servicios asociados a ellas, tuvieron un dinamismo que anuló o redujo fuertemente el de los demás sectores de la economía (Hora 2010). Estas consecuencias de las corrientes globales no son inevitables; hay ejemplos históricos de posicionamientos efectivos por parte de India, México y el Imperio Otomano (Williamson 2011). Por eso, Aldo Ferrer (1997) ha llamado a su aceptación acrítica la Visión Fundamentalista de la globalización. Pero caer en el otro extremo, negándola, no hará desaparecer los datos de la realidad internacional que están atrás de estas tendencias: los cambios en el transporte y las comunicaciones, el acelerado flujo de capitales, el aprovechamiento de la mano de obra a bajo costo. Para diseñar políticas públicas es más sensato tenerlas en cuenta, en principio para minimizar sus efectos negativos, e idealmente para usarlas a nuestro favor.

\section{Ignorar, o actuar como si se ignorase, la rica bibliografía relacionada con el manejo de conflictos, incluyendo los ambientales.}

En \#2 se enfatizó que las soluciones a los problemas ambientales son altamente dependientes del contexto político, económico y social. Sin embargo, esto no es equivalente a decir que lo pensado y experimentado en otros casos no pueda resultarnos útil. De otro modo, estaríamos condenados a la permanente improvisación, desaprovechando los cuerpos teóricos que existen acerca del manejo de los recursos. De los casi mil artículos catalogados por la base de datos SCOPUS desde 1972 que contienen los términos framework, resources, conflict, y management la mitad fueron publicados durante los últimos seis años (un síntoma del rápido crecimiento del tema es que Binder et al. 2013 han propuesto un esquemamarco... ipara sistematizar algunos de esos marcos!). Más arriba se mencionó la Tragedia de los Comunes, uno de los puntos de partida de lo que hoy se conoce como Segunda Ciencia Ambiental, que no sólo abarca lo biológico y ecológico sino también lo humano y cultural. Además de los aportes de Paul Sabatier, cabe señalar los de Elinor Ostrom (ver Dietz et al. 2003), Premio Nobel de Economía en 2009 por su análisis de las condiciones, sobre todo institucionales y de organización, necesarias para una buena gestión de los recursos compartidos. El énfasis está en la gobernanza, para alcanzar la cual un concepto crucial es el de Capital Social, que puede definirse como la conformación de lazos y redes de confianza. Éstas necesariamente requieren la aparición de líderes, muchas veces sin poder formal (Folke et al. 2005; McNie 2007) que, gracias a su prestigio e integridad, pueden canalizar el diálogo entre las partes antagónicas.

Un antecedente conocido para los ecólogos es el del Manejo Adaptativo, desarrollado a partir de la década del '70 (Holling et al. 1998), que ve la implementación de políticas o planes de manejo de recursos como experimentos a evaluar a través del seguimiento de sus consecuencias en el mundo real. Más cerca de las ciencias sociales, existe una variedad de esquemas y procesos de investigación-acción participativa de efectividad comprobada para facilitar la construcción de lazos más fuertes entre la investigación, la educación, y el uso de la información ambiental (Giller et al. 2008). Un ejemplo reciente al respecto es el taller organizado en la localidad de Rivadavia (cuenca del Salado, provincia de Buenos Aires) para discutir el tema de los excesos y déficits de agua a nivel municipal (Mercau et al. 2013).

\section{Ignorar, o actuar como si se ignorase, la necesidad del diálogo, en un proceso que nunca será breve, perfecto ni completo, pero que es la única vía democráticamente aceptable.}

Para algunos, el sistema de ciencia y técnica sería el encargado de que surjan opciones técnicamente sólidas y políticamente realistas a los problemas, dejando luego al proceso democrático la toma de decisiones. Éste sería un ejemplo de iniciativa desde la oferta, pero también hay muchos problemas que se plantean desde la demanda de la 
sociedad y sus dirigentes (Pielke 2007). Estas interacciones entre potenciales oferentes y demandantes de conocimiento no son fáciles, en parte por lo discutido en \#3, pero también por las barreras erigidas por el conocimiento tácito dentro de cada uno de esos dos dominios o culturas (Roux et al. 2006), y porque existen compromisos intrínsecos como, por ejemplo, entre la calidad de la información y la velocidad con que se la genera (García Fayos et al. 2008; Sarkki et al. 2013).

El elemento común a la mayoría de las propuestas para encarar estas dificultades es su énfasis en la necesidad de la co-producción de conocimiento entre los supuestos expertos y los usuarios (ver \#3). Los procesos participativos mencionados en \#8, además de sus beneficios directamente visibles tienen otro, en la medida que no se limiten a la mera comunicación o consulta unidireccional (McNie 2007; Giller et al. 2008): la gradual construcción de Capital Social. Combinando estas modalidades de trabajo con lo planteado en \#5 acerca del papel de los científicos y técnicos como brókers de alternativas, queda claro que las propuestas de innovación que deben entrar a la negociación pública no deben ser sólo técnicas, sino también institucionales y sociales.

\section{Pensar, o actuar como si se pensase, que existen soluciones definitivas a los problemas ambientales - como si no hubiese cambios en la técnica, la sociedad, y "co- adaptaciones".}

En \#4 se propuso un símil entre los problemas ambientales y los de la salud pública que aquí resulta nuevamente útil. Aspirar a que alguno de los dos pueda tener una solución definitiva es ilusorio: en parte porque tanto la ciencia médica como la ambiental avanzan, pero también porque ambos tipos de problemas dependen del contexto. Más aún: porque ese contexto incluye las respuestas de los actores involucrados a las decisiones de la sociedad. El dicho "hecha la ley, hecha la trampa" ilustra un caso simple y vinculado a lo legal, pero más en general debe decirse que las componentes de los sistemas socio-ecológicos co-evolucionan, no sólo en conocimiento y normativa, también en valores y formas de organización (su capital social), lo que retroalimenta su dinámica (Dietz et al. 2003).

\section{DISCUSIÓN}

Aquí se han presentado diez puntos entre los que hay argumentos tanto para lograr una visión irrealmente optimista de la situación

Tabla 1. Resumen del decálogo, con un nombre formal y otro coloquial para cada punto (y entre paréntesis una crítica abreviada que destaca su falsedad); el conjunto de los puntos 1, 2, 4, 7 y 10 representan una visión excesivamente optimista (y los 3, 5, 6 y 9, pesimista) de lo ambiental.

Table 1. Summary of the "ten ways", with a formal and an informal name for each one (and a critique between brackets); an excessively optimist view of environmental issues derives from considering only 1, 2, 4, 7 and 10, and the opposite from considering only 3, 5, 6 and 9).

\begin{tabular}{|c|c|c|}
\hline \# & $\begin{array}{l}\text { Problema: } \\
\text { actuar como si se pensase que... }\end{array}$ & $\begin{array}{l}\text { Nombre Coloquial } \\
\text { (y crítica) }\end{array}$ \\
\hline 1 & Simplismo, autorreferencia: lo ambiental es técnico & $\begin{array}{l}\text { Mi disciplina es la más relevante } \\
\text { (creerse único es poco original) }\end{array}$ \\
\hline 2 & $\begin{array}{l}\text { Cientificismo: el contexto político y socioeconómico no } \\
\text { importa }\end{array}$ & $\begin{array}{l}\text { ¡Vivan las tecnocracias!; está todo escrito* (cada caso/ } \\
\text { época difiere en algo importante) }\end{array}$ \\
\hline 3 & $\begin{array}{l}\text { Ignorancia y corrupción: el conocimiento siempre está } \\
\text { disponible }\end{array}$ & $\begin{array}{l}\text { Falta voluntad política } \\
\text { (los datos relevantes pueden no existir) }\end{array}$ \\
\hline 4 & $\begin{array}{l}\text { Anti-economicismo (I): hay soluciones sin costos de } \\
\text { oportunidad }\end{array}$ & $\begin{array}{l}\text { Lo deseable sólo tiene beneficios } \\
\text { (las panaceas no existen) }\end{array}$ \\
\hline 5 & $\begin{array}{l}\text { Uso selectivo de la información: hay unilateralidad } \\
\text { en los prejuicios }\end{array}$ & $\begin{array}{l}\text { Sólo los otros juegan sucio (todos tenemos ideología e } \\
\text { intereses) }\end{array}$ \\
\hline 6 & $\begin{array}{l}\text { Catastrofismo: la repetición de mensajes alarmistas es } \\
\text { útil }\end{array}$ & $\begin{array}{l}\text { El pastorcito que gritaba “ilobo, lobo!” } \\
\text { (poco creíble, cansa) }\end{array}$ \\
\hline 7 & Anti-economicismo (II): sirve negar la globalización & $\begin{array}{l}\text { ¿Qué importa lo que haga el resto? } \\
\text { (maldición de los recursos naturales) }\end{array}$ \\
\hline 8 & $\begin{array}{l}\text { Improvisación frente al conflicto: podemos reinventar } \\
\text { la rueda }\end{array}$ & $\begin{array}{l}\text { En la biblioteca no hay nada* } \\
\text { (Segunda Ciencia Ambiental) }\end{array}$ \\
\hline 9 & Menosprecio del diálogo racional y democrático & $\begin{array}{l}\text { Con ésos no se puede razonar* } \\
\text { (ni ingenuos ni descreídos a ultranza) }\end{array}$ \\
\hline 10 & $\begin{array}{l}\text { Voluntarismo: confianza en soluciones definitivas } \\
\text { y permanentes }\end{array}$ & $\begin{array}{l}\text { La cosa se va a arreglar (hay coevolución: hecha la ley, } \\
\text { hecha la trampa") }\end{array}$ \\
\hline
\end{tabular}


ambiental como una excesivamente pesimista (ver resumen en Tabla 1). Seleccionando los puntos $1,2,4,7$ y 10 , se puede argumentar que hay soluciones simples y definitivas, conocidas por todos ("nosotros"), y relativamente fáciles de implementar sin importar qué haga el resto del mundo. En el otro extremo, la conjunción de 3, 5, 6 y 9 conduce a afirmar que es inútil intentar dialogar porque "arriba no se juega limpio", no hay voluntad política, y estamos encaminados a una inevitable catástrofe. El punto 8, intrínsecamente malo ("no se sabe nada") es abiertamente contradictorio con el 2 ("está todo escrito"). Sin embargo, también es falso, por lo que no causa ningún problema en un Decálogo que estamos recomendando no usar.

Concentrarse en la versión más pesimista de los mensajes tiene un riesgo: la tentación de abroquelarse en la figura del activista o militante que denuncia pero que por principio no puede entablar un diálogo con el otro campo. El problema es que esto favorece el statu quo. Esta actitud de culpar "al sistema" desde afuera evita tener que participar, y el diálogo implica mucha preparación, trabajo y paciencia. El naturalista Edward O. Wilson insiste en que dejemos de ver los temas ambientales como si se tratasen de demandas especiales por parte de un grupo de interés y así contribuyamos a moverlos hacia el centro de las actividades humanas. Esto no significa subestimar el poder que tienen los grupos organizados para desacreditar la información ambiental (ver, por ejemplo, el documentado análisis de Jacques et al. (2008) acerca de las publicaciones ligadas a las usinas de ideas [think tanks] conservadoras). Por eso, los David militantes del campo ambiental tienen que dar combate contra los Goliat del mercado y la globalización eligiendo muy inteligentemente la estrategia, la ocasión y las armas. Estos diferirán según su contexto histórico e institucional, pero también según el tipo de problema y su escala espacial; e.g., con determinantes homogéneos planetariamente, como los gases de alto tiempo de residencia en la atmósfera, vs. los más localizados y regionales; ver Steffen \& Stafford Smith (2013).

De los puntos 4 y 7 , expresiones del antieconomicismo, surge que el comportamiento sostenible debe nada menos que resolver sus contradicciones con la sociedad de consumo, lo que de ningún modo está claro cómo debería hacerse, excepto por el acuerdogeneralizado de que el resultado debe tener en cuenta a los que menos tienen, quizá derivando en algún tipo de Economía Verde (Brito \& Stafford-Smith 2012). Sin entrar en el importantísimo debate de si los cambios requieren necesariamente nuevos paradigmas productivos o si pueden ser graduales, de todos modos es posible imaginar caminos que no involucran cambios revolucionarios del sistema. Un ejemplo es el importante papel que puede jugar, dentro de las reglas clásicas del mercado, la demanda selectiva ("comprar verde"). Esto es así porque los grupos con mayor poder adquisitivo son también los potencialmente mejor informados. En respuesta a estas tendencias de consumo, la oferta puede alinearse en iniciativas del tipo de las empresas Tipo B (www.bcorporation.net), que en su constitución establecen la simultánea sustentabilidad económica, social y ambiental, bajo los principios de gestión conocidos como Buenas Prácticas. Un aspecto crucial de las buenas prácticas podría ser cubierta dentro de los esquemas de cálculo de huellas (de carbono, energéticas y ecológicas en general), pero sólo si se analizan los procesos y sus consecuencias ambientales en sentido amplio (incluyendo su comparación con las opciones que reemplazan). Una trampa potencial de estos esquemas es que se dedique más esfuerzo a publicitar la supuesta "cara verde" de la empresa o producto (greenwashing) que a generar los cambios que efectivamente disminuyan los impactos indeseables. En todos estos temas, el papel de los profesionales del ambiente como docentes, consultores e investigadores es insustituible.

Aspirando a buscar generalidades, algunos puntos del decálogo se trataron de asimilar a conocidas falacias, es decir razonamientos incorrectos. Otra falacia, clasificable como negación del antecedente, es el Dilema del Progresista (Sarewitz 2010): si nos parece que la raíz de un problema ambiental está en una falla ética, parece insatisfactorio solucionarlo mediante una vía técnica o legal en lugar de hacerlo mediante cambios de comportamiento derivados de modificaciones profundas en las convicciones-como si estuviésemos más preocupados por las causas culturales de los problemas que por sus consecuencias prácticas. Estas requieren acciones que pueden parecer pedestres, como revisar la legislación y los detalles de los protocolos de aprobación de pesticidas, la capacitación técnica del personal que los usa, y la educación de los consumidores 
para que acepten alimentos con imperfecciones (tales como marcas externas sin consecuencias para la salud y que sólo podrían evitarse con aplicaciones más frecuentes o potentes). Nada evita que las transformaciones más profundas que requieren cambios actitudinales y plazos largos se hagan en paralelo con cambios técnicos y legales de resultado más rápido. Para algunos, quizá hasta un argumento como el de Steffen \& Stafford Smith (2013) acerca de la conveniencia para los ricos de una mayor redistribución de la riqueza, podría tener "gusto a poco".

La ya presentada falacia ad hominem suele estar acompañada de una variante conocida como Alegato Especial, que da a entender que el asunto en discusión tiene particularidades que el oponente no puede comprender debido a su falta de preparación, sutileza o empatía (una criollísima chicana). Este tipo de falacias pueden ser admisibles en el contexto de la retórica política donde, por ejemplo, la credibilidad de un dirigente o candidato sí es una cuestión relevante. Pero si empezamos a aceptarlas acríticamente, y a hacerlas habituales en nuestros escritos y clases, sólo estaremos contribuyendo a la confusión, deformando a los alumnos en lugar de hacerlos fructíferamente escépticos, y no haciéndole ningún favor a la agenda ambiental - la que en mi opinión deberíamos empezar a presentar de un modo cada vez menos épico, para instalarla como parte necesaria de los problemas del día a día de la sociedad.

Agradecimientos: A la Comisión Directiva de la ASAE por haberme honrado con la designación como miembro de la CATA. A R Berardo, C Cattaneo, A Cingolani, E Jobbágy, F Menvielle, J Paruelo, M Román y un revisor anónimo por sus comentarios y aportes de bibliografía. A mis compañeros de cátedra M Aguiar, M Oesterheld y M Semmartin, por el aliento a esta iniciativa. A los alumnos de Ecología, Impacto Ambiental, y el Taller de Problemáticas Ambientales de la FAUBA, por obligarme con sus preguntas y planteos a mantenerme siempre alerta a errores de razonamiento e interferencia de prejuicios (supuestos tácitos)-sobre todo los míos.

\section{BiBLIOGRAFÍA}

Bauman, Z. 2011. Daños Colaterales; Desigualdades Sociales en la Era Global. FCE, Buenos Aires.

Becher, T. 1991. Tribus y Territorios Académicos. Gedisa, Barcelona.

Binder, CR; J Hinkel; PWG Bots \& C PAHL-Wostl. 2013. Comparison of frameworks for analyzing socialecological systems. Ecology and Society, 18:26.

Brito, L \& M. StAFFord SMith (2012). The State of the Planet Declaration. Planet Under Pressure Conference.
International Council for Science, Londres.

Dietz, T; E Ostrom \& PC Stern. 2003. The struggle to govern the commons. Science, 302:1907-12.

FERrer, A. 1997. Hechos y Ficciones de la Globalización. Argentina y el MERCOSUR en el Sistema Internacional . FCE, Buenos Aires

Folke, C; T Hahn ; P. Olsson \& J Norberg. 2005. Adaptive governance of social-ecological systemas. Annu. Rev. Environ. Resour., 30:441-73.

García-Fayos, P; FJ Bonet; F Valladares; A Traveset; JG PAUSAS; ET AL. 2008. El ecólogo en su laberinto. Ecosistemas, 17:125-127.

GibBons, M; C Limoges; H Nowotny; S Schwartzman; P Scotт; M Trow. 1994. The New Production of Knowledge. Sage, London.

Giller, KE; C Leeuwis; JA Andersson; W Andriesse \& A. BROUWER; ET AL. 2008. Competing claims on natural resources: what role for science? Ecology and Society, 13:34.

GURVICH, DE; D RENISON \& F BARRI. 2009. El rol del ecólogo ante la crisis ambiental actual. Ecología Austral, 19: 233238.

Hardin, G. 1968. The tragedy of the commons. Science, 162:1243-1248.

Holling, CS; F Berkes \& C Folke. 1998. Science, sustainability and resource management. En: Berkes, F; C Folke (Eds.) Linking Social and Ecological Systems: Management Practices and Social Mechanisms for Building Resilience. Cambridge, Cambridge, MA.

HorA, R. 2010. Historia Económica Argentina en el Siglo XIX. Editorial Siglo XXI, Buenos Aires.

JACKSON, R. 2002. The Earth Remains Forever: Generations at a Crossroads. University of Texas Press, Austin.

Jacques, PJ; RE Dunlap \& M Freeman. 2008. The organisation of denial: Conservative think tanks and environmental scepticism. Environmental Politics, 17: 349-385.

JASANOFF, SS. 1990. The Fifth Branch: Science Advisors as Policy-makers. Harvard University Press, Cambridge.

JobbáGy EG \& FernándEZ RJ. 2012. Reseña del libro “The Honest Broker; Making Sense of Science in Policy and Politics", de R.A. Pielke Jr. Agronomía y Ambiente, 32:81-82.

Mercau, JL; EG Jobbágy; E Viglizzo; A Menendez; C Di BELLA; ET AL. 2013. Sequía e inundación en la hiperllanura pampeana. Una mirada desde el lote al municipio. Agronomía E Ambiente, 33: 71-77.

McNIE, EC. 2007. Reconciling the supply of scientific information with user demands: an analysis of the problem and review of the literature. Environmental Science and Policy, 10:17-38.

Paruelo, JM. 2009. En relación con el artículo “El rol del ecólogo ante la actual crisis ambiental", de Gurvich et al., en este número. Ecología Austral, 19: 255-258.

PIELKe, RA JR. 2007. The Honest Broker; Making Sense of Science in Policy and Politics. Cambridge University Press, NY.

Prebisch, R. 1950. The Economic Development of Latin America and Its Principal Problems. United Nations, NY.

Reynolds, JF; DM StafFord SMith; E LAMBin; BL TuRner II; M MORTIMORE; ET AL. 2007. Global desertificacion: building a science for dryland development. Science, 316: $847-851$.

RHODEs, R. 1987. The Making of the Atomic Bomb. Simon \& 
Schuster, N.Y.

Rockström, J; W Steffen; K Noone; Å Persson; FS Chapin III ET AL. 2009. Planetary boundaries: exploring the safe operating space for humanity. Ecology and Society, 14:32.

Román, M. 2013. Teoría económica y Ciencias ambientales. Un recorrido histórico: de los fisiócratas a los institucionalístas. Agronomía \& Ambiente, 33:57-70.

Roux, DJ; KH Rogers; HC Biggs; PJ Ashton \& A SERgeant. 2006. Bridging the science-management divide: moving from unidirectional knowledge transfer to knowledge interfacing and sharing. Ecology and Society, 11: 4.

Sabatier, PA \& MA Zafonte. 2001. Policy Knowledge: Advocacy Organizations. En: International Encyclopedia of the Social and Behavioral Sciences, 17:11563-11568. Elsevier, Amsterdam.

Sábato, JA. 2011. El Pensamiento Latinoamericano en la Problemática Ciencia-Tecnología-Desarrollo-Dependencia. Biblioteca Nacional, Buenos Aires.

SAREWITZ, D. 2004. How science makes environmental controversies worse. Environmental Science E Policy, 7:385-403.

SARewitZ, D. 2010. Political Effectiveness in Science and Technology. In: Carrier, M; A Nordmann (eds.), Science in the Context of Application. Springer, Dordrecht, pp 301-315.

SARKKI, S; J Niemela; R Tinch \& S VAN DEN Hove. 2013. Balancing credibility, relevance and legitimacy: A critical assessment of trade-offs in science-policy interfaces. Science and Public Policy, 41:194-206.

Steffen, W \& M Stafford Smith. 2013. Planetary boundaries, equity and global sustainability: why wealthy countries could benefit from more equity. Current Opinion in Environmental Sustainability, 5:1-6.

SVAmpa, M. 2013. Consenso de los commodities y lenguajes de valoración en América Latina. Nueva Sociedad, 244:30-46.

Varsavsky, O. 1969. Ciencia, Política y Cientificismo.Centro Editor de América Latina, Buenos Aires.

Williamson, JG. 2011 Trade and Poverty: When the Third World Fell Behind. MIT Press. Cambridge, MA. 